\title{
Subharmonics, Chaos, and Beyond
}

\author{
Laszlo Adler - Adler Consultants Inc. / Ohio State University \\ William T. Yost and John H. Cantrell - NASA-Langley Research Center
}

\begin{abstract}
While studying finite amplitude ultrasonic wave resonance in a one dimensional liquid-filled cavity formed by a narrow band transducer and a plane reflector, subharmonics of the driver's frequency were observed $(1,2)$ in addition to the expected harmonic structure. Subsequently, it was realized that the system was one of the many examples of parametric resonance in which the observed subharmonics are parametrically generated. The generation mechanism also requires a sufficiently high threshold value of the driving amplitude so that the system becomes increasingly nonlinear in response. The nonlinear features were recently investigated and are the focus of this paper. An ultrasonic interferometer with optical precision was built. The transducers were compressional, undamped quartz and Lithium Niobate crystals ranging from 1-10 $\mathrm{MHz}$, driven by a high power amplifier. Both an optical diffraction system and a receiver transducer attached to an aligned reflector were used to observe the generated frequency components in the cavity. There are at least 5 regions of excitation that were identified:

1. Linear region: at low intensity of the ultrasonic wave only the driving frequency component is present. The diffraction pattern of a light beam, normal to the sound field, is symmetric.

2. Nonlinear region: with increased sound amplitude the diffraction pattern becomes asymmetrical indicating the generation of harmonics.

3. Subharmonic region: with further increase of the amplitude above a threshold value (and sensitive to alignment of the transmitter and the reflector), subharmonics are generated which are indicated by additional orders in the diffraction pattern.

4. Chaos: at sufficiently high ultrasonic amplitude the diffraction pattern is smeared out. This region is identified as the Chaotic Region.

5. Beyond chaos: further increase of the amplitude produces a stable pattern beyond the chaotic region. The diffraction pattern is almost continuous indicating many low frequency components.

A first-principle-based explanation of the experimental findings is presented.
\end{abstract}

INTRODUCTION. It is well known that if a parameter of an oscillatory system varies periodically between certain limits, the system can also oscillate with a frequency roughly equal to one-half of the driving frequency. The term parametric excitation is used to describe such phenomena. The most quoted, readily observed, and understood problem was reported by Melde in 1859. Melde's experimental arrangement consisted of a stretched string of length $l$ and mass per unit length $\mu$ with one end fastened to the prong of a tuning fork. When the fork set the string into longitudinal vibration with amplitude A and frequency $2 \Omega$, Melde found that transverse vibrations of displacement amplitude g were excited with frequencies nearly half of that of the tuning fork. Lord Rayleigh in 1887 recognized that the subharmonic generation is the result of instabilities caused by variation of the tension $\mathrm{T}_{0}$ and showed that the Mathieu's equation

$$
\frac{d^{2} g}{d t^{2}}+\frac{T_{0}}{l^{2} \mu}(1-2 h \cos 2 \Omega t) g=0
$$

describes the main features of the problem; where $\mathrm{h}=\mathrm{A} / l$. Solutions of this equation are of the Floquet type. Recent analysis indicates (3) that Melde's experiment may also show chaotic behavior. Other examples of parametric phenomena in physics are discussed in ref (4). The objective of this paper is to discuss some features of an ultrasonic parametric system and to demonstrate the excitation of chaos and chaos reversal in the system.

ULTRASONIC PARAMETRIC GENERATOR. The ultrasonic parametric generator consists of a liquid filled one dimensional cavity bounded at one end by a rigid reflector and the other end by an oscillating boundary, a piezo-electric transducer. If the driver transducer amplitude is high, harmonics are generated by the nonlinearity of the medium. If the driver amplitude is then increased to a sufficiently larger value (and when accurate alignment of the transducer and reflector is achieved) other frequencies, called subharmonics, are generated.

A model was developed (5) which assumes that the subharmonics are parametrically excited waves produced by instabilities introduced through the vibration of the transducer face. The vibration of the transducer periodically alters the cavity length and therefore the resonant frequencies. The threshold for subharmonic generation depends on the amplitude of the oscillations. Parametric oscillation in a liquid filled cavity is given by the Mathieu-type expression 


$$
\frac{d^{2} g}{d z^{2}}+\frac{a \alpha c}{\omega} \frac{d g}{d z}+(a-2 q \cos z) g=0
$$

where $\mathrm{g}$ is the cavity displacement, $\alpha$ is the wave attenuation coefficient, $\mathrm{c}$ is the sound velocity, $\omega$ is the angular frequency, $\mathrm{a}=\left(\omega_{\mathrm{n}} / \omega\right)^{2}$ where $\omega$ is the nth resonance frequency, $\mathrm{q}=\mathrm{a}(\mathrm{A} / l)$ where $\mathrm{A}$ is the tranducer drive displacement amplitude, $l$ is the cavity length, and $\mathrm{z}=\omega \mathrm{t}$ where $\mathrm{t}$ is time. The conditions for threshold subharmonic excitation are obtained from equation (2). There are two conditions which must be satisfied:

1. The resonant frequency of the lowest subharmonic mode must be nearly equal to one half the drive frequency;

2. The amplitude A of the driver must be large enough to satisfy the threshold condition given as

$$
A>l \alpha c / \omega \text {. }
$$

The solutions of the Mathieu's equation occur in the alternating regions or bands of stability and instability (defined by the factors "a" and "q" in Eq.(2)) with increasing values of the factor "a" (see Fig. 1).

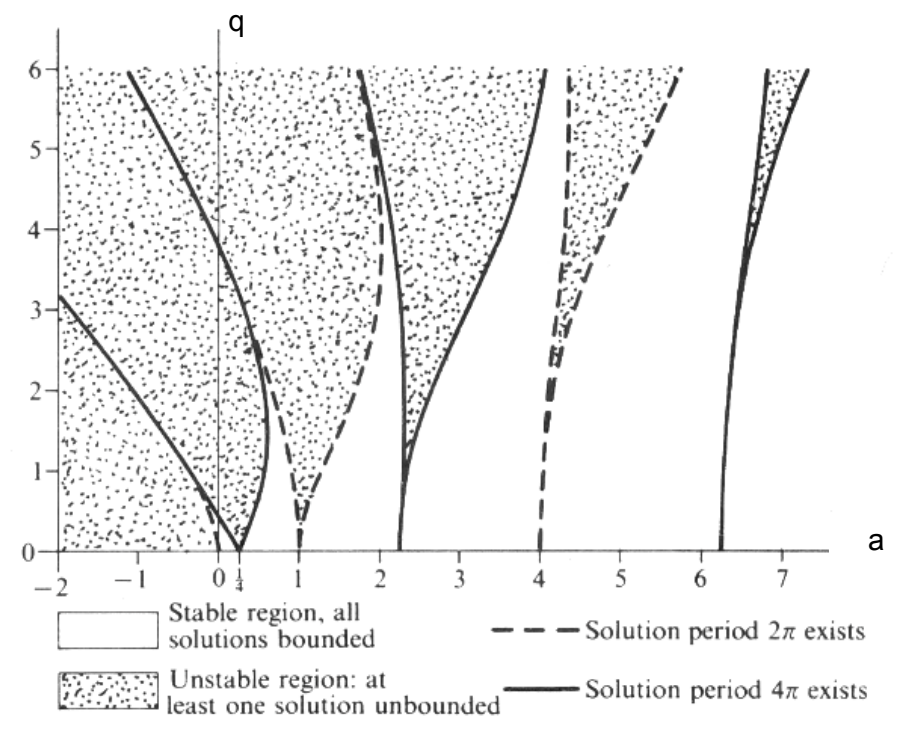

FIGURE 1. Regions of solution for the Mathieu's Function

Both a and $\mathrm{q}$ are functions of the transducer drive amplitude. A more complete accounting of the phenomenon may be described by expanding Eq.(2) to include nonlinear contributions. The more complete equation is given as (5)

$$
\frac{d^{2} g}{d z^{2}}+\frac{a \alpha c}{\omega} \frac{d g}{d z}+(a-2 q \cos z) g+\frac{\omega^{2}}{2 c^{2}}\left(\frac{B}{A}+2\right)\left(\frac{B}{A}+1\right)\left(\frac{a}{2}-2 q \cos 2 z\right) g^{3}=0
$$

where B/A is the nonlinearity parameter of the liquid. A first order approximation to the solution of Eq.(4) indicates that the threshold condition of parametric excitation is the same as that given by equation (2). Other theoretical studies to address the nonlinear features of this parametric system have been reported recently $(6,7)$.

\section{REGIONS OF ULTRASONIC WAVE GENERATION IN THE LIQUID FILLED}

CAVITY. The experimental system shown in Fig. 2 consists of an interferometer with optical precision controls used to adjust the positions of the piezo-electric transducer $(1 \mathrm{MHz}-10 \mathrm{MHz}$; driven by a powerful amplifier) and a receiving transducer attached to an aligned reflector with lapped flat and parallel surfaces used to measure the generated frequency components in the cavity.

A visual assessment of the phenomena is obtained by passing laser light through the ultrasonic beam as indicated in Fig. 3. The laser light is diffracted into various orders $n$ at angles $\alpha_{n}$ given by

$$
\sin \left(\alpha_{n}\right)=n \lambda \omega / c
$$




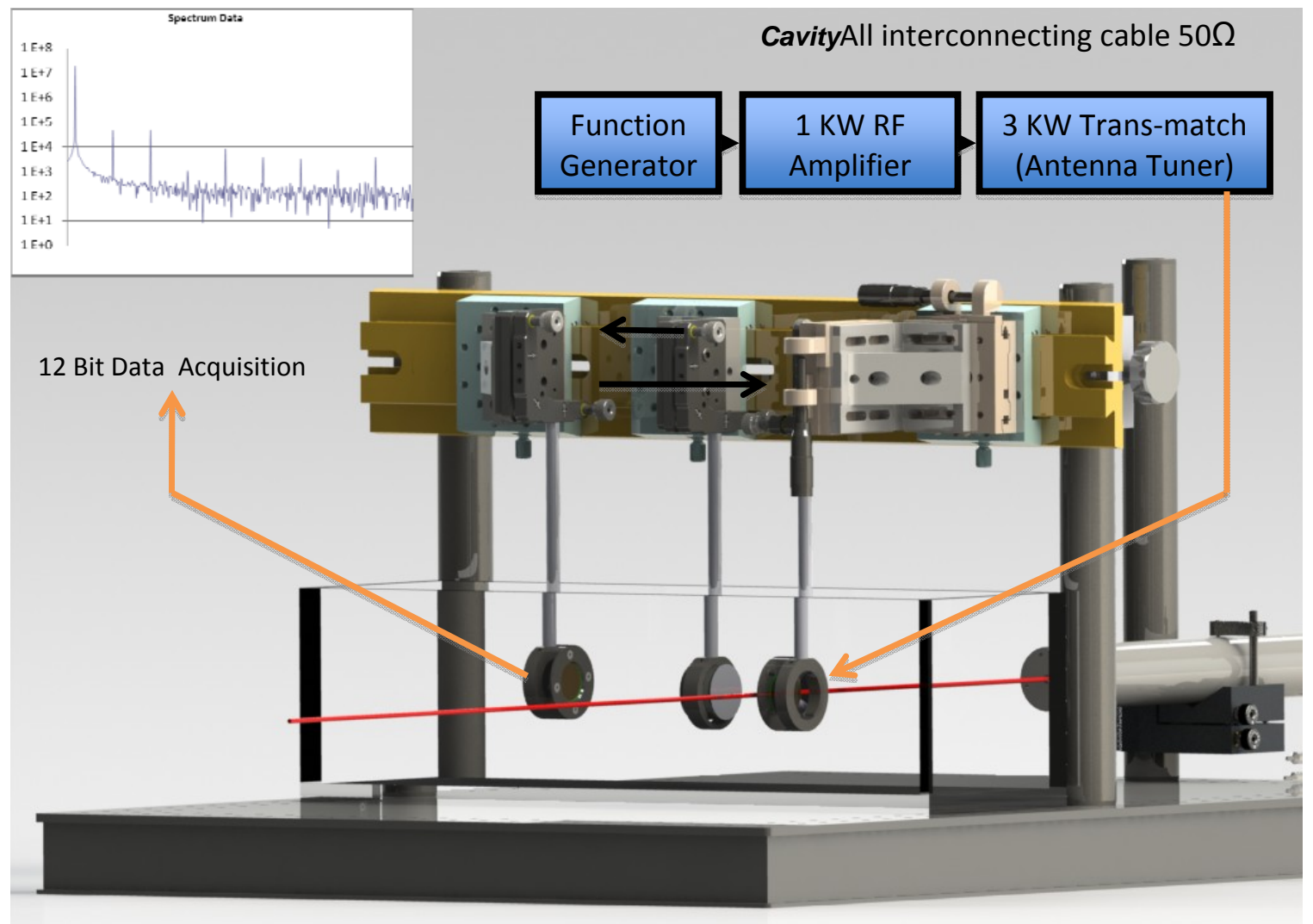

FIGURE 2. Experimental system

where $\lambda$ is the wavelength of the light. Fig. 3 shows the diffraction patterns obtained for various transducer drive amplitudes (voltages): (a) low amplitude ultrasonic waves (5V); (b) finite amplitude waves (50V) resulting in an asymmetric diffraction pattern; and (c) parametric resonance (150V) producing extra diffraction orders due to the generation of subharmonics. The received frequency spectrum corresponding to the parametric resonance region is shown in Fig. 4. In addition to the driver transducer frequency at $5 \mathrm{MHz}$, the second and third harmonics at $10 \mathrm{MHz}$ and $15 \mathrm{MHz}$, as well as subharmonics at $2.5 \mathrm{MHz}$ are displayed in Fig. 4.

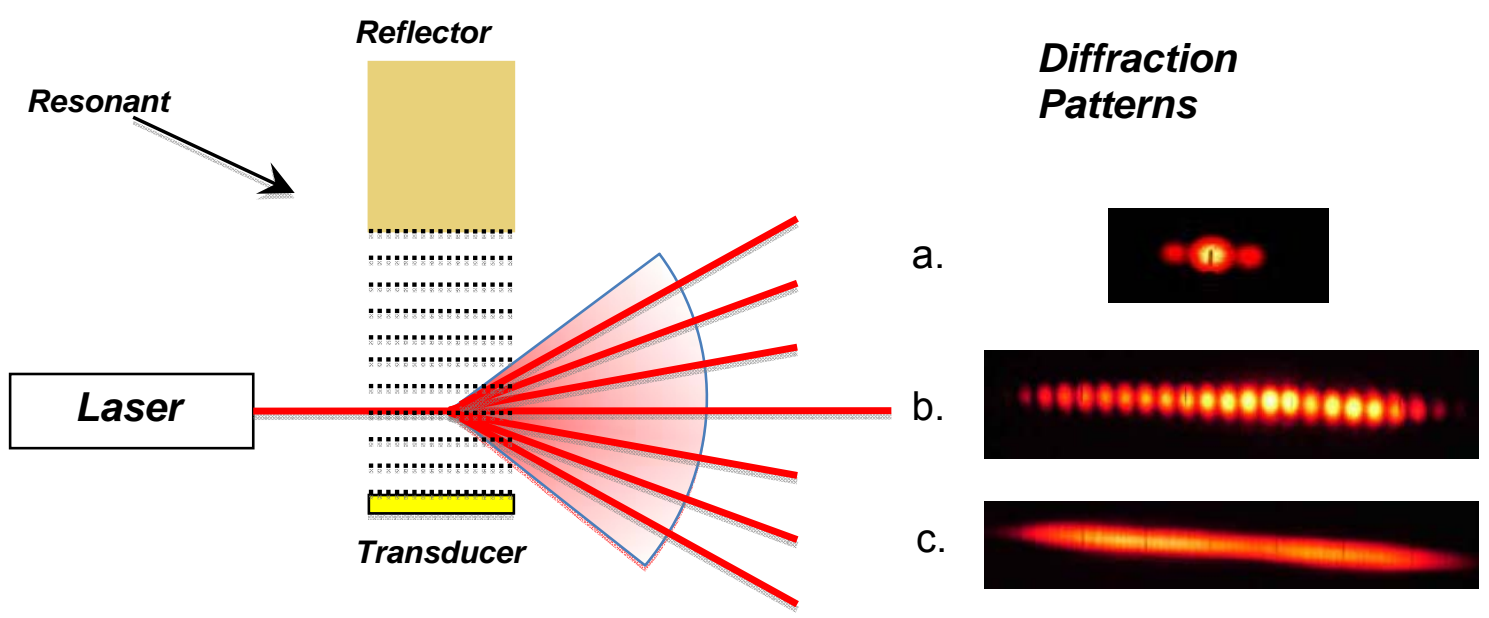

FIGURE 3. Laser beam diffraction by ultrasonic wave: a. Linear Region, b. Nonlinear Region, c. Subharmonic Region 


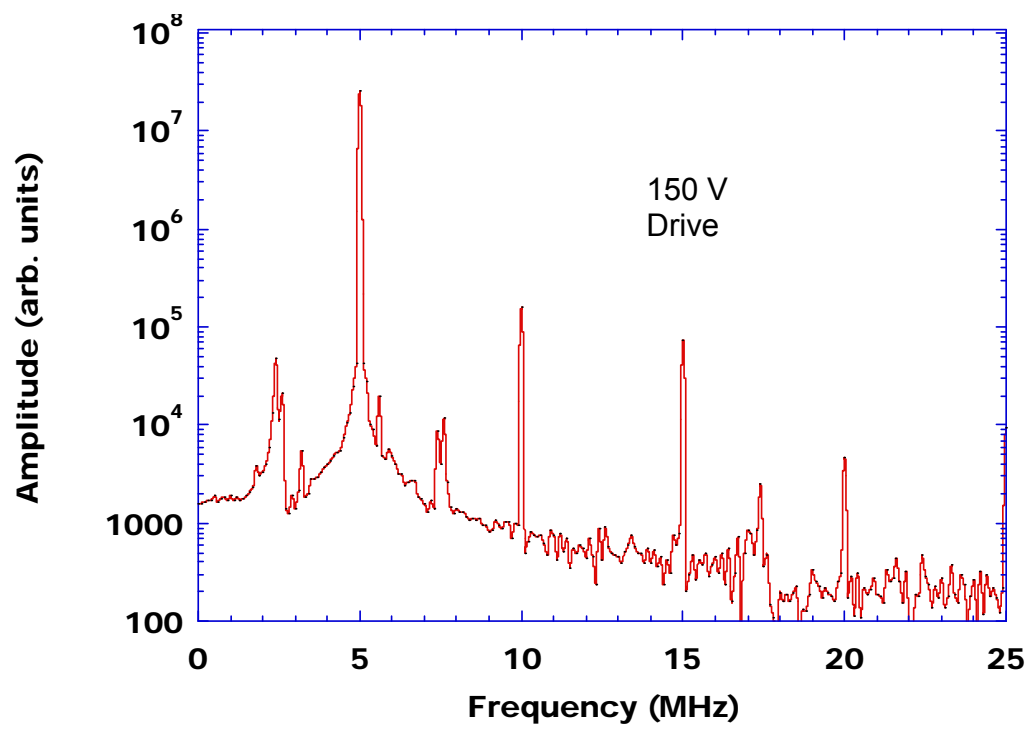

FIGURE 4. A plot of wave amplitudes vs. Frequency components including cavity generated Fractional Harmonics

PATH TO CHAOS. A significantly higher transducer drive voltage (450V) in the parametric resonance region leads to a cascade of bifurcations with increasing drive amplitudes that culminate in the generation of the chaotic pattern shown in Fig. 5a. Instead of distinct diffraction orders, the laser produces a smeared out image due to the chaotic oscillations. Further increases in the transducer drive voltage (to 500V) leads to a second region of stability following the region of chaotic instability. The diffraction pattern in the second region of stability is shown on Fig. 5b. The pattern is similar to that of Fig. 3c, indicating the presence of stable subharmonics.

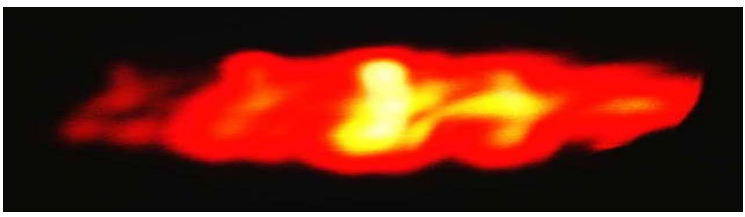

a. Chaos

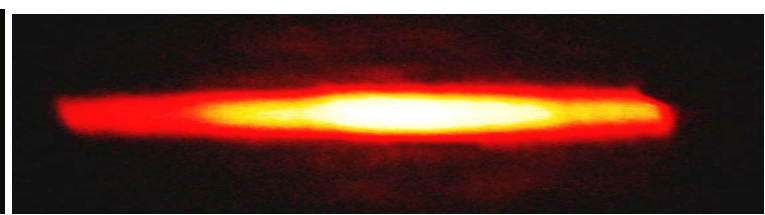

b. Beyond Chaos

FIGURE 5. Laser Beam Diffraction Ultrasonic Waves: a. In the Chaotic Region (450V) b. Beyond Chaotic Region (500V).

CONCLUSIONS. In an ultrasonic parametric system, increasing acoustic drive amplitudes from a region of oscillation stability into an unstable region leads to a cascade of bifurcations (subharmonics) culminating in chaotic oscillations. A further increase in the amplitude results in a reversion of the chaos into a second region of stability.

\section{REFERENCES}

1. Korpel , A. and Adler, R. "Parametric Phenomena Observed on Ultrasonic Waves in Water" Appl. Phys. Lett. 7, 106 (1965)

2. Adler, L. and Breazeale, M. A. "Excitation of Subharmonics in a Resonant Ultrasonic Wave System” Die Naturwissenschaften 55, 385 (1968)

3.Tufillaro, B. "Nonlinear and Chaotic Vibrations". Am.J. Phys. 57. 5. 408 (1989)

4. Adler, L. and Breazeale, M.A. "Parametric Phenomena in Physics” Am. J. Phys. 39.1522 (1971)

5.Adler, L. and Breazeale, M.A. "Generation of Fractional Harmonics in Resonant Ultrasonic Wave System” J. Acoust. Soc. Am. 46, 5. 1077 (1970)

6. Perez-Arjona, I., Sanchez-Moroillo, V.J. and Espinosa,V. "Bistable and Dynamic States of Parametrically Excited Ultrasonic Waves in a Fluid- Filled Interferometer” J. Acoust. Soc. Am. 125, 6. 3555 (2009) 
7. Goldberg, Z., Goldberg, I. and Goldberg, A. "Two Regimes of the Parametrically Self-Exciting Ultrasonic Standing Waves” J. Acoust. Soc. Am. 129, 6. 3483 (2011)

This work is supported by the Vehicle Systems Safety Technologies (VSST) Program, at NASA Langley Research Center. 